\title{
Even and odd $q$-deformed charge coherent states and their nonclassical properties
}

\author{
X.-M. Liu ${ }^{a, b}$, C. Quesne ${ }^{b, *}$ \\ a Department of Physics, Beijing Normal University, Beijing 100875, China \\ b Physique Nucléaire Théorique et Physique Mathématique, \\ Université Libre de Bruxelles, Campus de la Plaine CP229, \\ Boulevard du Triomphe, B-1050 Brussels, Belgium ${ }^{1}$
}

\begin{abstract}
Even and odd $q$-deformed charge coherent states are constructed, their (over)completeness proved and their generation explored. A $D$-algebra realization of the $\mathrm{SU}_{q}(1,1)$ generators is given in terms of them. They are shown to exhibit $\mathrm{SU}_{q}(1,1)$ squeezing and two-mode $q$-antibunching, but neither one-mode, nor two-mode $q$-squeezing.
\end{abstract}

Keywords: Even/odd charge coherent states; q-deformation; Completeness relations; Squeezing

PACS number(s): 42.50.Dv; 03.65.-W; 03.65.Ca; 03.65.Fd

* Corresponding author

E-mail addresses: liuxm@263.net (X.-M. Liu), cquesne@ulb.ac.be (C. Quesne).

${ }^{1}$ Mailing address 


\section{Introduction}

The coherent states introduced by Schrödinger [1] and Glauber [2] are the eigenstates of the boson annihilation operator, and have widespread applications in the fields of physics [3-7]. However, in all the cases the quanta involved are uncharged. In 1976, Bhaumik et al. $[4,8]$ constructed the boson coherent states which, carrying definite charge, are the eigenstates of both the pair boson annihilation operator and the charge operator. This kind of states are the so-called charge coherent states. Based on this work, the charge coherent states for $\mathrm{SU}(2)[9,10]$, $\mathrm{SU}(3)$ [11], and arbitrary compact Lie groups [12] were also put forward.

The concept of charge coherent states has proved to be very useful in many areas, such as elementary particle physics [13-17], quantum field theory [12,18,19], nuclear

physics [20], thermodynamics [21-23], quantum mechanics [24], and quantum optics [25-27]. Moreover, some schemes for generating charge coherent states in quantum optics were proposed $[25,26,28,29]$.

As is well known, the even and odd coherent states [30], which are the two orthonormalized eigenstates of the square of the boson annihilation operator, play an important role in quantum optics [31-33]. In a previous Letter [34], inspired by the above idea, one of the authors (X.-M.L.) has generalized the charge coherent states to the even and odd charge coherent states, defined as the two orthonormalized eigenstates of both the square of the pair boson annihilation operator and the charge operator.

On the other hand, quantum groups [35,36], introduced as a mathematical description of deformed Lie algebras, have given the possibility of generalizing the notion 
of coherent states to the case of $q$-deformations [37-41]. A q-deformed harmonic oscillator $[37,42]$ was defined in terms of $q$-boson annihilation and creation operators, the latter satisfying the quantum Heisenberg-Weyl algebra $[37,42,43]$, which plays an important role in quantum groups. The $q$-deformed coherent states introduced by Biedenharn [37] are the eigenstates of the $q$-boson annihilation operator. Such states have been well studied $[38,39,44,45]$, and widely applied to quantum optics and mathematical physics [41,46-50]. Furthermore, the $q$-deformed charge coherent states $[51,52]$ were constructed as the eigenstates of both the pair $q$-boson annihilation operator and the charge operator.

A natural extension of the $q$-deformed coherent states is provided by the even and odd $q$-deformed coherent states [53], which are the two orthonormalized eigenstates of the square of the $q$-boson annihilation operator. In a parallel way, it is very desirable to generalize the $q$-deformed charge coherent states to the even and odd ones, defined as the two orthonormalized eigenstates of both the square of the pair $q$-boson annihilation operator and the charge operator.

This Letter is arranged as follows. In Section 2, the even and odd $q$-deformed charge coherent states are constructed. Their completeness is proved in Section 3. Section 4 is devoted to generating them. In Section 5 , they are used to provide a $D$ algebra realization of the $\mathrm{SU}_{q}(1,1)$ generators. Their nonclassical properties, such as $\mathrm{SU}_{q}(1,1)$ squeezing, single- or two-mode $q$-squeezing, and two-mode $q$-antibunching, are studied in Section 6. Section 7 contains a summary of the results. 


\section{Even and odd $q$-deformed charge coherent states}

Two mutually commuting $q$-deformed harmonic oscillators are defined in terms of two pairs of independent $q$-boson annihilation and creation operators $a_{i}, a_{i}^{+}$ $(i=1,2)$, together with corresponding number operators $N_{i}$, satisfying the quantum Heisenberg-Weyl algebra

$$
\begin{gathered}
a_{i} a_{i}^{+}-q a_{i}^{+} a_{i}=q^{-N_{i}}, \\
{\left[N_{i}, a_{i}^{+}\right]=a_{i}^{+}, \quad\left[N_{i}, a_{i}\right]=-a_{i},}
\end{gathered}
$$

where $q$ is a positive real deformation parameter. The operators $a_{i}, a_{i}^{+}$, and $N_{i}$ act in the Fock space with basis $|n\rangle_{i}(n=0,1,2, \ldots)$, such that

$$
a_{i}|0\rangle_{i}=0, \quad|n\rangle_{i}=\frac{\left(a_{i}^{+}\right)^{n}}{\sqrt{[n] !}}|0\rangle_{i}
$$

where

$$
\begin{gathered}
{[n] ! \equiv[n]_{q} !=[n]_{q}[n-1]_{q} \ldots[1]_{q}, \quad[0] !=1} \\
{[n]_{q}=\frac{q^{n}-q^{-n}}{q-q^{-1}} \equiv[n] .}
\end{gathered}
$$

Their action on the basis states is given by

$$
a_{i}|n\rangle_{i}=\sqrt{[n]}|n-1\rangle_{i}, \quad a_{i}^{+}|n\rangle_{i}=\sqrt{[n+1]}|n+1\rangle_{i}, \quad N_{i}|n\rangle_{i}=n|n\rangle_{i} .
$$

Note that $[n]$ is invariant under $q \leftrightarrow 1 / q$. We may therefore choose $0<q \leq 1$. In the following, $[n]$ will refer to the $q$-deformed $n$ defined by (5) corresponding to the base $q$. If the base is different, then it will be indicated explicitly.

The $q$-boson operators $a_{i}$ and $a_{i}^{+}$can be constructed from the conventional boson annihilation and creation operators $b_{i}, b_{i}^{+}$in the following way [54]:

$$
a_{i}=\sqrt{\frac{\left[N_{i}+1\right]}{N_{i}+1}} b_{i}, \quad a_{i}^{+}=b_{i}^{+} \sqrt{\frac{\left[N_{i}+1\right]}{N_{i}+1}}
$$


where $N_{i}=b_{i}^{+} b_{i}$. It is worth noticing that $\left[N_{i}\right]=a_{i}^{+} a_{i}$.

We first briefly review the $q$-deformed charge coherent states. The operators $a_{1}\left(a_{1}^{+}\right)$and $a_{2}\left(a_{2}^{+}\right)$are assigned the "charge" quanta 1 and -1 , respectively. Thus the charge operator is given by

$$
Q=N_{1}-N_{2}
$$

In view of the fact that

$$
\left[Q, a_{1} a_{2}\right]=0
$$

the $q$-deformed charge coherent states are defined as the eigenstates of both the pair $q$-boson annihilation operator $a_{1} a_{2}$ and the charge operator $Q$, i.e.,

$$
a_{1} a_{2}|\xi, \underline{q}\rangle=\xi|\xi, \underline{q}\rangle, \quad Q|\xi, \underline{q}\rangle=\underline{q}|\xi, \underline{q}\rangle,
$$

where $\xi$ is a complex number and $\underline{q}$ is the charge number, which is a fixed integer. With the help of the two-mode Fock space's completeness relation

$$
\sum_{m=0}^{\infty} \sum_{n=0}^{\infty}|m, n\rangle\langle m, n|=I, \quad| m, n\rangle \equiv|m\rangle_{1}|n\rangle_{2}
$$

where $|m\rangle_{1}$ and $|n\rangle_{2}$ are the eigenstates of $N_{1}$ and $N_{2}$ corresponding to the eigenvalues $m$ and $n$, respectively, the $q$-deformed charge coherent states can be expanded as

$$
\begin{aligned}
|\xi, \underline{q}\rangle & =N_{\underline{q}} \sum_{p=\max (0,-\underline{q})}^{\infty} \frac{\xi^{p+\min (0, \underline{q})}}{\{[p] ![p+\underline{q}] !\}^{1 / 2}}|p+\underline{q}, p\rangle \\
& =\left\{\begin{array}{l}
N_{\underline{q}} \sum_{n=0}^{\infty} \frac{\xi^{n}}{\{[n] ![n+\underline{q}]\}^{1 / 2}}|n+\underline{q}, n\rangle, \quad \underline{q} \geq 0, \\
N_{\underline{q}} \sum_{n=0}^{\infty} \frac{\xi^{n}}{\{[n] ![n-\underline{q}]\}^{1 / 2}}|n, n-\underline{q}\rangle, \quad \underline{q} \leq 0,
\end{array}\right.
\end{aligned}
$$

where the normalization factor $N_{\underline{q}}$ is given by

$$
N_{\underline{q}} \equiv N_{\underline{q}}\left(|\xi|^{2}\right)=\left\{\sum_{n=0}^{\infty} \frac{\left(|\xi|^{2}\right)^{n}}{[n] ![n+|\underline{q}|] !}\right\}^{-1 / 2} .
$$


We now seek the even and odd $q$-deformed charge coherent states, which are the two orthonormalized eigenstates of both the square $\left(a_{1} a_{2}\right)^{2}$ and the operator $Q$, i.e.,

$$
\left(a_{1} a_{2}\right)^{2}|\xi, \underline{q}\rangle_{e(o)}=\xi^{2}|\xi, \underline{q}\rangle_{e(o)}, \quad Q|\xi, \underline{q}\rangle_{e(o)}=\underline{q}|\xi, \underline{q}\rangle_{e(o)}, \quad{ }_{e}\langle\xi, \underline{q} \mid \xi, \underline{q}\rangle_{o}=0 .
$$

The solutions to equation (14) are

$$
\begin{gathered}
|\xi, \underline{q}\rangle_{e}=N_{\underline{q}}^{e} \sum_{p=\max (0,-\underline{q} / 2)}^{\infty} \frac{\xi^{2 p+\min (0, \underline{q})}}{\{[2 p] ![2 p+\underline{q}] !\}^{1 / 2}}|2 p+\underline{q}, 2 p\rangle \\
=\left\{\begin{array}{l}
N_{\underline{q}}^{e} \sum_{n=0}^{\infty} \frac{\xi^{2 n}}{\{[2 n] ![2 n+\underline{q}] !\}^{1 / 2}}|2 n+\underline{q}, 2 n\rangle, \quad \underline{q} \geq 0, \\
N_{\underline{q}}^{e} \sum_{n=0}^{\infty} \frac{\xi^{2 n}}{\{[2 n] ![2 n-\underline{q}] !\}^{1 / 2}}|2 n, 2 n-\underline{q}\rangle, \quad \underline{q} \leq 0,
\end{array}\right. \\
|\xi, \underline{q}\rangle_{o}=N_{\underline{q}}^{o} \sum_{p=\max (0,-\underline{q} / 2)}^{\infty} \frac{\xi^{2 p+1+\min (0, \underline{q})}}{\{[2 p+1] ![2 p+1+\underline{q}] !\}^{1 / 2}}|2 p+1+\underline{q}, 2 p+1\rangle \\
=\left\{\begin{array}{c}
N_{\underline{q}}^{o} \sum_{n=0}^{\infty} \frac{\xi^{2 n+1}}{\{[2 n+1] ![2 n+1+q] !\}^{1 / 2}}|2 n+1+\underline{q}, 2 n+1\rangle, \quad \underline{q} \geq 0, \\
N_{\underline{q}}^{o} \sum_{n=0}^{\infty} \frac{\xi^{2 n+1}}{\{[2 n+1] ![2 n+1-q] !\}^{1 / 2}}|2 n+1,2 n+1-\underline{q}\rangle, \quad \underline{q} \leq 0,
\end{array}\right.
\end{gathered}
$$

where the normalization factors $N_{\underline{q}}^{e, o}$ are given by

$$
\begin{gathered}
N_{\underline{q}}^{e} \equiv N_{\underline{q}}^{e}\left(|\xi|^{2}\right)=\left\{\sum_{n=0}^{\infty} \frac{\left(|\xi|^{2}\right)^{2 n}}{[2 n] ![2 n+|\underline{q}|] !}\right\}^{-1 / 2}, \\
N_{\underline{q}}^{o} \equiv N_{\underline{q}}^{o}\left(|\xi|^{2}\right)=\left\{\sum_{n=0}^{\infty} \frac{\left(|\xi|^{2}\right)^{2 n+1}}{[2 n+1] ![2 n+1+|\underline{q}|] !}\right\}^{-1 / 2} .
\end{gathered}
$$

In the limit $q \rightarrow 1$, they reduce to the usual even and odd charge coherent states constructed in Ref. [34]. Note that $|\xi, \underline{q}\rangle_{e(o)}$ are not eigenstates of $a_{1} a_{2}$.

From (15) and (16), it follows that

$$
\begin{aligned}
& e(o)\left\langle\xi, \underline{q} \mid \xi^{\prime}, \underline{q}^{\prime}\right\rangle_{e(o)}=N_{\underline{q}}^{e(o)}\left(|\xi|^{2}\right) N_{\underline{q}}^{e(o)}\left(\left|\xi^{\prime}\right|^{2}\right)\left[N_{\underline{q}}^{e(o)}\left(\xi^{*} \xi^{\prime}\right)\right]^{-2} \delta_{\underline{q}, \underline{q}^{\prime}}, \\
& e\left\langle\xi, \underline{q} \mid \xi^{\prime}, \underline{q}^{\prime}\right\rangle_{o}=0 .
\end{aligned}
$$

This further indicates that the even (odd) q-deformed charge coherent states are orthogonal with respect to the charge number $\underline{q}$ and that arbitrary even and odd 
$q$-deformed charge coherent states are orthogonal to each other. However, these even (odd) states are nonorthogonal with respect to the parameter $\xi$.

For the mean values of the operators $N_{1}$ and $N_{2}$, there exists the relation

$$
{ }_{e(o)}\left\langle\xi, \underline{q}\left|N_{1}\right| \xi, \underline{q}\right\rangle_{e(o)}=\underline{q}+{ }_{e(o)}\left\langle\xi, \underline{q}\left|N_{2}\right| \xi, \underline{q}\right\rangle_{e(o)} .
$$

In terms of the even and odd $q$-deformed charge coherent states, the $q$-deformed charge coherent states can be expanded as

$$
|\xi, \underline{q}\rangle=N_{\underline{q}}\left[\left(N_{\underline{q}}^{e}\right)^{-1}|\xi, \underline{q}\rangle_{e}+\left(N_{\underline{q}}^{o}\right)^{-1}|\xi, \underline{q}\rangle_{o}\right]
$$

where the normalization factors are such that

$$
N_{\underline{q}}^{-2}=\left(N_{\underline{q}}^{e}\right)^{-2}+\left(N_{\underline{q}}^{o}\right)^{-2}
$$

\section{Completeness of even and odd $q$-deformed charge coherent states}

Let us begin with some $q$-deformed formulas which are useful in the proof of completeness of the even and odd $q$-deformed charge coherent states. The $q$-deformed Bessel function of (integer) order $\nu$ may be defined by [55]

$$
J_{\nu}(q, x)=\sum_{k=0}^{\infty} \frac{(-1)^{k}}{[k] ![\nu+k] !}\left(\frac{x}{\sqrt{q}[2]_{\sqrt{q}}}\right)^{\nu+2 k},
$$

where $[n]_{\sqrt{q}}$ is defined as in equation (5) except for replacing $q$ by $\sqrt{q}$. An integral representation of the $q$-deformed modified Bessel function of order $\nu$ is given by [56]

$$
K_{\nu}(q, x)=\frac{1}{[2]_{\sqrt{q}}}\left(\frac{x}{[2]_{\sqrt{q}}}\right)^{\nu} \int_{0}^{\infty} d_{q} t \frac{1}{t^{\nu+1}} e_{q}(-t) e_{q}\left(-\frac{x^{2}}{\left([2]_{\sqrt{q}}\right)^{2} t}\right),
$$

where $d_{q} t$ is a standard $q$-integration $[44,57,58]$, and $e_{q}(x)$ is a $q$-exponential function [44]

$$
e_{q}(x)= \begin{cases}\sum_{n=0}^{\infty} \frac{x^{n}}{[n] !}, & \text { for } x>-\zeta \\ 0, & \text { otherwise }\end{cases}
$$


with $-\zeta$ being the largest zero of $e_{q}(x)$. Then, it follows that [56]

$$
\int_{0}^{\infty} d_{\sqrt{q}} u u^{2 p+\nu+1} K_{\nu}\left(q,[2]_{\sqrt{q}} u\right)=\frac{[\nu+p] ![p] !}{\left([2]_{\sqrt{q}}\right)^{2}} .
$$

We now prove that the even and odd $q$-deformed charge coherent states form an (over)complete set, that is to say

$$
\sum_{\underline{q}=-\infty}^{\infty} \int \frac{d_{q}^{2} \xi}{\pi} \phi_{\underline{q}}(\xi) N_{\underline{q}}^{2}\left[\left(N_{\underline{q}}^{e}\right)^{-2}|\xi, \underline{q}\rangle_{e} e\left\langle\xi, \underline{q}\left|+\left(N_{\underline{q}}^{o}\right)^{-2}\right| \xi, \underline{q}\right\rangle_{o o}\langle\xi, \underline{q}|\right] \equiv \sum_{\underline{q}=-\infty}^{\infty} I_{\underline{q}}=I
$$

where

$$
d_{q}^{2} \xi=|\xi| d_{\sqrt{q}}|\xi| d \theta, \quad \xi=|\xi| e^{i \theta}
$$

and

$$
\phi_{\underline{q}}(\xi)=\frac{\left([2]_{\sqrt{q}}\right)^{2}}{2}(-\mathrm{i}) \underline{q} J_{\underline{q}}\left(q, \mathrm{i} \sqrt{q}[2]_{\sqrt{q}}|\xi|\right) K_{\underline{q}}\left(q,[2]_{\sqrt{q}}|\xi|\right) .
$$

Note that the integral over $\theta$ is a standard integration while that over $|\xi|$ is a $q$ integration.

In fact, for $\underline{q} \geq 0$, we have

$$
\begin{aligned}
& I_{\underline{q}}=\int \frac{d_{q}^{2} \xi}{\pi} \phi_{\underline{q}}(\xi) N_{\underline{q}}^{2} \sum_{j=0}^{1} \sum_{n, m} \frac{\xi^{2 n+j} \xi^{* 2 m+j}|2 n+j+\underline{q}, 2 n+j\rangle\langle 2 m+j+\underline{q}, 2 m+j|}{\{[2 n+j] ![2 n+j+\underline{q}] ![2 m+j] ![2 m+j+\underline{q}] !\}^{1 / 2}} \\
& =\int_{0}^{\infty} \frac{d_{\sqrt{q}}|\xi|}{\pi} \frac{\left([2]_{\sqrt{q}}\right)^{2}}{2}|\xi|^{\underline{q}+1} K_{\underline{q}}\left(q,[2]_{\sqrt{q}}|\xi|\right) \sum_{j=0}^{1} \sum_{n, m}|\xi|^{2(n+m+j)} \int_{-\pi}^{\pi} d \theta e^{2 \mathrm{i}(n-m) \theta} \\
& \times \frac{|2 n+j+\underline{q}, 2 n+j\rangle\langle 2 m+j+\underline{q}, 2 m+j|}{\{[2 n+j] ![2 n+j+\underline{q}] ![2 m+j] ![2 m+j+\underline{q}] !\}^{1 / 2}} \\
& =\int_{0}^{\infty} d_{\sqrt{q}}|\xi|\left([2]_{\sqrt{q}}\right)^{2}|\xi|^{q+1} K_{\underline{q}}\left(q,[2]_{\sqrt{q}}|\xi|\right) \\
& \times \sum_{j=0}^{1} \sum_{n=0}^{\infty} \frac{\left(|\xi|^{2}\right)^{2 n+j}|2 n+j+\underline{q}, 2 n+j\rangle\langle 2 n+j+\underline{q}, 2 n+j|}{[2 n+j] ![2 n+j+\underline{q}] !} \\
& =\sum_{n=0}^{\infty} \frac{\left([2]_{\sqrt{q}}\right)^{2}}{[n] ![n+\underline{q}] !}|n+\underline{q}, n\rangle\left\langle n+\underline{q},\left.n\left|\int_{0}^{\infty} d_{\sqrt{q}}\right| \xi|| \xi\right|^{2 n+\underline{q}+1} K_{\underline{q}}\left(q,[2]_{\sqrt{q}}|\xi|\right)\right. \\
& =\sum_{n=0}^{\infty}|n+\underline{q}, n\rangle\langle n+\underline{q}, n| \text {. }
\end{aligned}
$$


Similarly, for $\underline{q} \leq 0$, we get

$$
I_{\underline{q}}=\sum_{n=0}^{\infty}|n, n-\underline{q}\rangle\langle n, n-\underline{q}| .
$$

Consequently, we derive

$$
\begin{aligned}
\sum_{\underline{q}=-\infty}^{\infty} I_{\underline{q}} & =\sum_{n=0}^{\infty}\left(\sum_{\underline{q}=-\infty}^{-1}|n, n-\underline{q}\rangle\left\langle n, n-\underline{q}\left|+\sum_{\underline{q}=0}^{\infty}\right| n+\underline{q}, n\right\rangle\langle n+\underline{q}, n|\right) \\
& =\sum_{m=0}^{\infty} \sum_{n=0}^{\infty}|m, n\rangle\langle m, n|=I .
\end{aligned}
$$

Hence, the even and odd $q$-deformed charge coherent states are qualified to make up an overcomplete representation. It should be mentioned that $I_{\underline{q}}$ represents the resolution of unity in the subspace where $Q=\underline{q}$.

\section{Generation of even and odd $q$-deformed charge coherent states}

By means of (12), (15) and (16), we find

$$
\begin{aligned}
|\xi, \underline{q}\rangle_{e} & =\frac{1}{2} \frac{N_{\underline{q}}^{e}}{N_{\underline{q}}}(|\xi, \underline{q}\rangle+|-\xi, \underline{q}\rangle), \\
|\xi, \underline{q}\rangle_{o} & =\frac{1}{2} \frac{N_{\underline{q}}^{o}}{N_{\underline{q}}}(|\xi, \underline{q}\rangle-|-\xi, \underline{q}\rangle) .
\end{aligned}
$$

This shows that the even (odd) $q$-deformed charge coherent states can be generated by the symmetric (antisymmetric) combination of $q$-deformed charge coherent states as the charge is conserved. This is similar to the case of even (odd) $q$-deformed coherent states, which are combinations of $q$-deformed coherent states, namely,

$$
\begin{aligned}
|\xi\rangle_{e} & =\frac{1}{2} \frac{N^{e}}{N}(|\xi\rangle+|-\xi\rangle), \\
|\xi\rangle_{o} & =\frac{1}{2} \frac{N^{o}}{N}(|\xi\rangle-|-\xi\rangle),
\end{aligned}
$$


where

$$
\begin{gathered}
|\xi\rangle=N \sum_{n=0}^{\infty} \frac{\xi^{n}}{\sqrt{[n] !}}|n\rangle, \\
N=e_{q}^{-1 / 2}\left(|\xi|^{2}\right), \quad N^{e} \equiv N^{e}\left(|\xi|^{2}\right)=\cosh _{q}^{-1 / 2}|\xi|^{2}, \quad N^{o} \equiv N^{o}\left(|\xi|^{2}\right)=\sinh _{q}^{-1 / 2}|\xi|^{2},
\end{gathered}
$$

with

$$
\begin{aligned}
& \cosh _{q} x=\sum_{n=0}^{\infty} \frac{x^{2 n}}{[2 n] !} \\
& \sinh _{q} x=\sum_{n=0}^{\infty} \frac{x^{2 n+1}}{[2 n+1] !} .
\end{aligned}
$$

The even (odd) q-deformed charge coherent states can also be obtained from the states $(36)-(38)$ according to the following expression

$$
\begin{aligned}
& |\xi, \underline{q}\rangle_{e(o)} \\
& \quad=\left\{\begin{array}{l}
N_{\underline{q}}^{e(o)} e_{q}^{1 / 2}\left(\left|\xi_{1}\right|^{2}\right)\left[N^{e(o)}\left(\left|\xi_{2}\right|^{2}\right)\right]^{-1} \xi_{1}-\underline{q} \int_{-\pi}^{\pi} \frac{d \alpha}{2 \pi} e^{+\mathrm{i} q} \alpha\left|e^{-\mathrm{i} \alpha} \xi_{1}\right\rangle \otimes\left|e^{\mathrm{i} \alpha} \xi_{2}\right\rangle_{e(o)}, \quad \underline{q} \geq 0, \\
N_{\underline{q}}^{e(o)} e_{q}^{1 / 2}\left(\left|\xi_{1}\right|^{2}\right)\left[N^{e(o)}\left(\left|\xi_{2}\right|^{2}\right)\right]^{-1} \xi_{1}+\underline{q} \int_{-\pi}^{\pi} \frac{d \alpha}{2 \pi} e^{-\mathrm{i} q} \underline{q}\left|e^{\mathrm{i} \alpha} \xi_{2}\right\rangle_{e(o)} \otimes\left|e^{-\mathrm{i} \alpha} \xi_{1}\right\rangle, \quad \underline{q} \leq 0,
\end{array}\right.
\end{aligned}
$$

where $\xi=\xi_{1} \xi_{2}$. Such a representation is very useful since the properties of $q$-deformed coherent states and even (odd) $q$-deformed coherent states can now be employed in a study of the properties of even (odd) q-deformed charge coherent states. The expression for the latter given in (42) has a very simple group-theoretical interpretation: in (42) one suitably averages over the U(1)-group (caused by the charge operator $Q$ ) action on the product of $q$-deformed coherent states and even (odd) $q$-deformed coherent states, which then projects out the $Q=\underline{q}$ charge subspace contribution.

It is easy to see that in the limit $q \rightarrow 1$, the above discussion gives back the corresponding results for the usual even and odd charge coherent states obtained in Ref. [34]. 


\section{D-algebra realization of $\mathrm{SU}_{q}(1,1)$ generators}

As is well known, the coherent state $D$-algebra $[6,59]$ is a mapping of quantum observables onto a differential form that acts on the parameter space of coherent states, and has a beautiful application in the reformulation of the entire laser theory in terms of $C$-number differential equations [60]. We shall construct the $D$-algebra realization of the $q$-deformed $\mathrm{SU}_{q}(1,1)$ generators corresponding to the unnormalized even and odd $q$-deformed charge coherent states, defined by

$$
\left.\| \underline{q}\rangle_{e(o)} \equiv \| \xi, \underline{q}\right\rangle_{e(o)}=\left[N_{\underline{q}}^{e(o)}\right]^{-1}|\xi, \underline{q}\rangle_{e(o)} .
$$

The action of the operators $a_{i}, a_{i}^{+}$and $N_{i}$ on the column vector composed of $\left.\| \underline{q}\right\rangle_{e}$ and $\| \underline{q}\rangle_{o}$ can be written in the matrix form:

Positive $Q$

$a_{1}\left[\begin{array}{l}\| \underline{q}\rangle_{e} \\ \| \underline{q}\rangle_{o}\end{array}\right]=\left[\begin{array}{l}\| \underline{q}-1\rangle_{e} \\ \| \underline{q}-1\rangle_{o}\end{array}\right]$

$a_{2}\left[\begin{array}{l}\| \underline{q}\rangle_{e} \\ \| \underline{q}\rangle_{o}\end{array}\right]=\xi\left[\begin{array}{ll}0 & 1 \\ 1 & 0\end{array}\right]\left[\begin{array}{l}\| \underline{q}+1\rangle_{e} \\ \| \underline{q}+1\rangle_{o}\end{array}\right]$,

$a_{1}^{+}\left[\begin{array}{c}\| \underline{q}\rangle_{e} \\ \| \underline{q}\rangle_{o}\end{array}\right]=\xi^{-\underline{q}} \frac{d}{d_{q} \xi} \xi^{\underline{q}+1}\left[\begin{array}{l}\| \underline{q}+1\rangle_{e} \\ \| \underline{q}+1\rangle_{o}\end{array}\right]$,

$a_{2}^{+}\left[\begin{array}{l}\| \underline{q}\rangle_{e} \\ \| \underline{q}\rangle_{o}\end{array}\right]=\frac{d}{d_{q} \xi}\left[\begin{array}{cc}0 & 1 \\ 1 & 0\end{array}\right]\left[\begin{array}{l}\| \underline{q}-1\rangle_{e} \\ \| \underline{q}-1\rangle_{o}\end{array}\right]$,

$N_{1}\left[\begin{array}{l}\| \underline{q}\rangle_{e} \\ \| \underline{q}\rangle_{o}\end{array}\right]=\left(\xi \frac{d}{d \xi}+\underline{q}\right)\left[\begin{array}{c}\| \underline{q}\rangle_{e} \\ \| \underline{q}\rangle_{o}\end{array}\right]$,

$N_{2}\left[\begin{array}{c}\| \underline{q}\rangle_{e} \\ \| \underline{q}\rangle_{o}\end{array}\right]=\xi \frac{d}{d \xi}\left[\begin{array}{c}\| \underline{q}\rangle_{e} \\ \| \underline{q}\rangle_{o}\end{array}\right]$,
Negative $Q$

$a_{1}\left[\begin{array}{l}\| \underline{q}\rangle_{e} \\ \| \underline{q}\rangle_{o}\end{array}\right]=\xi\left[\begin{array}{ll}0 & 1 \\ 1 & 0\end{array}\right]\left[\begin{array}{l}\| \underline{q}-1\rangle_{e} \\ \| \underline{q}-1\rangle_{o}\end{array}\right]$,

$a_{2}\left[\begin{array}{l}\| \underline{q}\rangle_{e} \\ \| \underline{q}\rangle_{o}\end{array}\right]=\left[\begin{array}{l}\| \underline{q}+1\rangle_{e} \\ \| \underline{q}+1\rangle_{o}\end{array}\right]$,

$a_{1}^{+}\left[\begin{array}{l}\| \underline{q}\rangle_{e} \\ \| \underline{q}\rangle_{o}\end{array}\right]=\frac{d}{d_{q} \xi}\left[\begin{array}{cc}0 & 1 \\ 1 & 0\end{array}\right]\left[\begin{array}{l}\| \underline{q}+1\rangle_{e} \\ \| \underline{q}+1\rangle_{o}\end{array}\right]$,

$a_{2}^{+}\left[\begin{array}{l}\| \underline{q}\rangle_{e} \\ \| \underline{q}\rangle_{o}\end{array}\right]=\xi \frac{q}{d_{q} \xi} \xi^{-\underline{q}+1}\left[\begin{array}{l}\| \underline{q}-1\rangle_{e} \\ \| \underline{q}-1\rangle_{o}\end{array}\right]$,

$N_{1}\left[\begin{array}{l}\| \underline{q}\rangle_{e} \\ \| \underline{q}\rangle_{o}\end{array}\right]=\xi \frac{d}{d \xi}\left[\begin{array}{l}\| \underline{q}\rangle_{e} \\ \| \underline{q}\rangle_{o}\end{array}\right]$,

$N_{2}\left[\begin{array}{l}\| \underline{q}\rangle_{e} \\ \| \underline{q}\rangle_{o}\end{array}\right]=\left(\xi \frac{d}{d \xi}-\underline{q}\right)\left[\begin{array}{l}\| \underline{q}\rangle_{e} \\ \| \underline{q}\rangle_{o}\end{array}\right]$,

where $d / d \xi$ is a standard differential operator, whereas $d / d_{q} \xi$ is a $q$-differential one $[39,44,58]$, defined by

$$
\frac{d}{d_{q} \xi} f(x)=\frac{f(q x)-f\left(q^{-1} x\right)}{q x-q^{-1} x} .
$$


The $q$-deformed $\mathrm{SU}_{q}(1,1)$ algebra consists of three generators $K_{0}, K_{+}$, and $K_{-}$, satisfying the commutation relations

$$
\left[K_{+}, K_{-}\right]=-\left[2 K_{0}\right], \quad\left[K_{0}, K_{ \pm}\right]= \pm K_{ \pm}
$$

and is realized in terms of the two-mode $q$-boson operators as

$$
K_{-}=a_{1} a_{2}, \quad K_{+}=a_{1}^{+} a_{2}^{+}, \quad K_{0}=\frac{1}{2}\left(N_{1}+N_{2}+1\right) .
$$

Actually, the even and odd $q$-deformed charge coherent states are also the eigenstates of the square of $K_{-}$.

The $D$-algebra of the $\mathrm{SU}_{q}(1,1)$ generators $A$ may be defined for the action on the ket coherent states (43) or for that on the corresponding bras as

$$
\begin{aligned}
& A\left[\begin{array}{l}
\| \underline{q}\rangle_{e} \\
\| \underline{q}\rangle_{o}
\end{array}\right]=D^{k}(A)\left[\begin{array}{l}
\| \underline{q}\rangle_{e} \\
\| \underline{q}\rangle_{o}
\end{array}\right], \\
& {\left[\begin{array}{c}
e\langle\underline{q} \| \\
o\langle\underline{q} \|
\end{array}\right] A=D^{b}(A)\left[\begin{array}{c}
e\langle\underline{q} \| \\
o\langle\underline{q} \|
\end{array}\right],}
\end{aligned}
$$

respectively. Using (44) and (47), we get for the former

$$
\begin{aligned}
D^{k}\left(K_{-}\right) & =\xi\left[\begin{array}{ll}
0 & 1 \\
1 & 0
\end{array}\right], \\
D^{k}\left(K_{+}\right) & =\xi^{-|\underline{q}|} \frac{d}{d_{q} \xi} \xi^{|\underline{q}|+1} \frac{d}{d_{q} \xi}\left[\begin{array}{ll}
0 & 1 \\
1 & 0
\end{array}\right], \\
D^{k}\left(K_{0}\right) & =\frac{1}{2}\left(2 \xi \frac{d}{d \xi}+|\underline{q}|+1\right) I,
\end{aligned}
$$

while the latter can be obtained from the adjoint relation

$$
D^{b}(A)=\left[D^{k}\left(A^{+}\right)\right]^{*}
$$

Thus, the $D$-algebra of the $\mathrm{SU}_{q}(1,1)$ generators corresponding to the unnormalized even and odd $q$-deformed charge coherent states has been realized in a $q$-differentialoperator matrix form. 
From (48) and (50), we clearly see that the unnormalized even and odd $q$-deformed charge coherent states can be transformed into each other by the action of the operator $a_{1} a_{2}$. Actually, $a_{1} a_{2}$ plays the role of a connecting operator between the two kinds of states.

It is easy to check that in the limit $q \rightarrow 1$, the above discussion gives back that carried out in Ref. [34] for the usual even and odd charge coherent states.

\section{Nonclassical properties of even and odd q- deformed charge coherent states}

In this section, we will study some nonclassical properties of the even and odd $q$-deformed charge coherent states, such as $\mathrm{SU}_{q}(1,1)$ squeezing, single- or two-mode $q$-squeezing, and two-mode $q$-antibunching.

\section{1. $\mathrm{SU}_{q}(1,1)$ squeezing}

In analogy with the definition of $\mathrm{SU}(1,1)$ squeezing [61], we introduce $\mathrm{SU}_{q}(1,1)$ squeezing in terms of the Hermitian q-deformed quadrature operators

$$
X_{1}=\frac{K_{+}+K_{-}}{2}, \quad X_{2}=\frac{\mathrm{i}\left(K_{+}-K_{-}\right)}{2}
$$

which satisfy the commutation relation

$$
\left[X_{1}, X_{2}\right]=\frac{\mathrm{i}}{2}\left[2 K_{0}\right]
$$

and the uncertainty relation

$$
\left\langle\left(\Delta X_{1}\right)^{2}\right\rangle\left\langle\left(\Delta X_{2}\right)^{2}\right\rangle \geq \frac{1}{16}\left|\left\langle\left[2 K_{0}\right]\right\rangle\right|^{2}
$$


A state is said to be $\mathrm{SU}_{q}(1,1)$ squeezed if

$$
\left\langle\left(\Delta X_{i}\right)^{2}\right\rangle<\frac{1}{4}\left|\left\langle\left[2 K_{0}\right]\right\rangle\right| \quad(i=1 \text { or } 2)
$$

Let us now calculate the fluctuations (variances) of $X_{1}$ and $X_{2}$ with respect to the even and odd $q$-deformed charge coherent states. Using (47) - (50) and (53), we get

$$
\begin{aligned}
{ }_{e}\left\langle\xi, \underline{q}\left|K_{+} K_{-}\right| \xi, \underline{q}\right\rangle_{e} & =|\xi|^{2} \overline{\tanh }_{\underline{q}}|\xi|^{2}, \\
{ }_{o}\left\langle\xi, \underline{q}\left|K_{+} K_{-}\right| \xi, \underline{q}\right\rangle_{o} & =|\xi|^{2} \overline{\operatorname{coth}}_{\underline{q}}|\xi|^{2},
\end{aligned}
$$

where

$$
\overline{\tanh }_{\underline{q}}|\xi|^{2} \equiv \frac{\overline{\sinh }_{\underline{q}}|\xi|^{2}}{\overline{\cosh }_{\underline{q}}|\xi|^{2}}, \quad \overline{\operatorname{coth}}_{\underline{q}}|\xi|^{2} \equiv \frac{1}{\overline{\tanh }_{\underline{q}}|\xi|^{2}}
$$

with

$$
\overline{\sinh }_{\underline{q}} x \equiv\left[N_{\underline{q}}^{o}(x)\right]^{-2}, \quad \overline{\cosh }_{\underline{q}} x \equiv\left[N_{\underline{q}}^{e}(x)\right]^{-2} .
$$

Furthermore,

$$
{ }_{e(o)}\left\langle K_{+}\right\rangle_{e(o)}={ }_{e(o)}\left\langle K_{-}\right\rangle_{e(o)}=0 .
$$

Thus, the fluctuations are given by

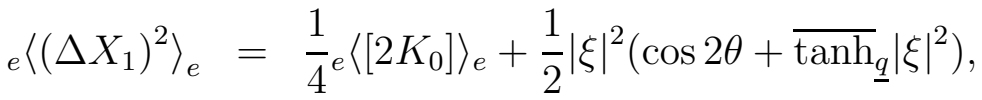

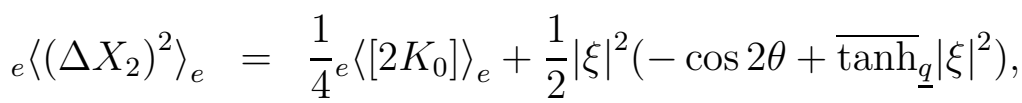

$$
\begin{aligned}
& { }_{o}\left\langle\left(\Delta X_{1}\right)^{2}\right\rangle_{o}=\frac{1}{4}{ }_{o}\left\langle\left[2 K_{0}\right]\right\rangle_{o}+\frac{1}{2}|\xi|^{2}\left(\cos 2 \theta+\overline{\operatorname{coth}}_{\underline{q}}|\xi|^{2}\right), \\
& { }_{o}\left\langle\left(\Delta X_{2}\right)^{2}\right\rangle_{o}=\frac{1}{4}{ }_{o}\left\langle\left[2 K_{0}\right]\right\rangle_{o}+\frac{1}{2}|\xi|^{2}\left(-\cos 2 \theta+\overline{\operatorname{coth}}_{\underline{q}}|\xi|^{2}\right) .
\end{aligned}
$$

According to $(63)-(66), \mathrm{SU}_{q}(1,1)$ squeezing of the states $|\xi, \underline{q}\rangle_{e(o)}$ occurs as long as

$$
\begin{aligned}
& \pm \cos 2 \theta+\overline{\tanh }_{\underline{q}}|\xi|^{2}<0, \\
& \pm \cos 2 \theta+\overline{\operatorname{coth}}_{\underline{q}}|\xi|^{2}<0,
\end{aligned}
$$


for one of the sign choices. Choose $\theta$ to be $\frac{\pi}{2}$ or 0 , so that $\pm \cos 2 \theta=-1$. When $|\xi| \leq 1, \overline{\tanh }_{\underline{q}}|\xi|^{2}<1$. Thus, condition (67) can be satisfied for $|\xi| \leq 1$. Using (17), (18), (23), (60), and (61), we can prove that

$$
\overline{\operatorname{coth}_{\underline{q}}}|\xi|^{2}=1+\frac{1}{\left.|\xi|^{|q|}\right|_{\sinh _{\underline{q}}}|\xi|^{2}} J_{|\underline{q}|}\left(q, \sqrt{q}[2]_{\sqrt{q}}|\xi|\right) .
$$

We know that $\overline{\sinh }_{\underline{q}}|\xi|^{2} \geq 0$. Obviously, $\overline{\operatorname{coth}_{\underline{q}}}|\xi|^{2}<1$ if $J_{|\underline{q}|}\left(q, \sqrt{q}[2]_{\sqrt{q}}|\xi|\right)<0$. In fact, for arbitrary fixed values of $\underline{q}$ and $q$, there surely exists some range of $|\xi|$ values such that $J_{|\underline{q}|}\left(q, \sqrt{q}[2]_{\sqrt{q}}|\xi|\right)<0$. For instance, numerical calculations show that, for $\underline{q}=0$ and $q=0.2, J_{0}\left(0.2, \sqrt{0.2}[2]_{\sqrt{0.2}}|\xi|\right)<0$ when $1.020 \leq|\xi| \leq 5.208$; for $\underline{q}= \pm 1$ and $q=0.5, J_{1}\left(0.5, \sqrt{0.5}[2]_{\sqrt{0.5}}|\xi|\right)<0$ when $1.808 \leq|\xi| \leq 3.770$; for $\underline{q}= \pm 2$ and $q=0.9, J_{2}\left(0.9, \sqrt{0.9}[2]_{\sqrt{0.9}}|\xi|\right)<0$ when $2.560 \leq|\xi| \leq 4.166$. Thus, for arbitrary fixed values of $\underline{q}$ and $q$, condition (68) can also be satisfied over some limited range of $|\xi|$ values.

From the above discussion, it is apparent that both even and odd $q$-deformed charge coherent states can exhibit $\mathrm{SU}_{q}(1,1)$ squeezing.

It is easy to verify that the $q$-deformed charge coherent states satisfy the equality in (56) and that $\left\langle\left(\Delta X_{1}\right)^{2}\right\rangle=\left\langle\left(\Delta X_{2}\right)^{2}\right\rangle$. Therefore, the $q$-deformed charge coherent states, contrary to the even and odd ones, are not $\mathrm{SU}_{q}(1,1)$ squeezed.

\subsection{Single-mode $q$-squeezing}

In analogy with the definition of single-mode squeezing [27], we introduce singlemode $q$-squeezing in terms of the Hermitian $q$-deformed quadrature operators for the individual modes

$$
Y_{1}=\frac{a_{1}^{+}+a_{1}}{2}, \quad Y_{2}=\frac{\mathrm{i}\left(a_{1}^{+}-a_{1}\right)}{2}
$$




$$
Z_{1}=\frac{a_{2}^{+}+a_{2}}{2}, \quad Z_{2}=\frac{\mathrm{i}\left(a_{2}^{+}-a_{2}\right)}{2}
$$

which satisfy the commutation relations

$$
\left[Y_{1}, Y_{2}\right]=\frac{\mathrm{i}}{2}\left[a_{1}, a_{1}^{+}\right], \quad\left[Z_{1}, Z_{2}\right]=\frac{\mathrm{i}}{2}\left[a_{2}, a_{2}^{+}\right]
$$

and the uncertainty relations

$$
\left\langle\left(\Delta Y_{1}\right)^{2}\right\rangle\left\langle\left(\Delta Y_{2}\right)^{2}\right\rangle \geq \frac{1}{16}\left|\left\langle\left[a_{1}, a_{1}^{+}\right]\right\rangle\right|^{2}, \quad\left\langle\left(\Delta Z_{1}\right)^{2}\right\rangle\left\langle\left(\Delta Z_{2}\right)^{2}\right\rangle \geq \frac{1}{16}\left|\left\langle\left[a_{2}, a_{2}^{+}\right]\right\rangle\right|^{2} .
$$

A state is said to be single-mode $q$-squeezed if

$$
\left\langle\left(\Delta Y_{i}\right)^{2}\right\rangle<\frac{1}{4}\left|\left\langle\left[a_{1}, a_{1}^{+}\right]\right\rangle\right|, \quad\left\langle\left(\Delta Z_{i}\right)^{2}\right\rangle<\frac{1}{4}\left|\left\langle\left[a_{2}, a_{2}^{+}\right]\right\rangle\right| \quad(i=1 \text { or } 2) .
$$

For the even and odd $q$-deformed charge coherent states, it always follows that

$$
{ }_{e(o)}\left\langle a_{1}\right\rangle_{e(o)}={ }_{e(o)}\left\langle a_{2}\right\rangle_{e(o)}={ }_{e(o)}\left\langle a_{1}^{2}\right\rangle_{e(o)}={ }_{e(o)}\left\langle a_{2}^{2}\right\rangle_{e(o)}={ }_{e(o)}\left\langle a_{1}^{+} a_{2}\right\rangle_{e(o)}=0
$$

Thus, the fluctuations are given by

$$
\begin{aligned}
e_{e(o)}\left\langle\left(\Delta Y_{1}\right)^{2}\right\rangle_{e(o)}={ }_{e(o)}\left\langle\left(\Delta Y_{2}\right)^{2}\right\rangle_{e(o)} & =\frac{1}{4}\left(e(o)\left\langle\left[a_{1}, a_{1}^{+}\right]\right\rangle_{e(o)}+2_{e(o)}\left\langle a_{1}^{+} a_{1}\right\rangle_{e(o)}\right) \\
& >\frac{1}{4} e(o)\left\langle\left[a_{1}, a_{1}^{+}\right]\right\rangle_{e(o)}, \\
{ }_{e(o)}\left\langle\left(\Delta Z_{1}\right)^{2}\right\rangle_{e(o)}=_{e(o)}\left\langle\left(\Delta Z_{2}\right)^{2}\right\rangle_{e(o)} & =\frac{1}{4}\left(e(o)\left\langle\left[a_{2}, a_{2}^{+}\right]\right\rangle_{e(o)}+2_{e(o)}\left\langle a_{2}^{+} a_{2}\right\rangle_{e(o)}\right) \\
& >\frac{1}{4} e(o)\left\langle\left[a_{2}, a_{2}^{+}\right]\right\rangle_{e(o)} .
\end{aligned}
$$

This shows that there is no single-mode $q$-squeezing in both even and odd $q$-deformed charge coherent states. The same situation occurs for the $q$-deformed charge coherent states $[62]$. 


\subsection{Two-mode $q$-squeezing}

In analogy with the definition of two-mode squeezing [63], we introduce two-mode $q$-squeezing in terms of the Hermitian $q$-deformed quadrature operators for the two modes

$W_{1}=\frac{Y_{1}+Z_{1}}{\sqrt{2}}=\frac{1}{\sqrt{8}}\left(a_{1}^{+}+a_{2}^{+}+a_{1}+a_{2}\right), \quad W_{2}=\frac{Y_{2}+Z_{2}}{\sqrt{2}}=\frac{\mathrm{i}}{\sqrt{8}}\left(a_{1}^{+}+a_{2}^{+}-a_{1}-a_{2}\right)$,

which satisfy the commutation relation

$$
\left[W_{1}, W_{2}\right]=\frac{1}{4} \mathrm{i}\left\{\left[a_{1}, a_{1}^{+}\right]+\left[a_{2}, a_{2}^{+}\right]\right\}
$$

and the uncertainty relation

$$
\left\langle\left(\Delta W_{1}\right)^{2}\right\rangle\left\langle\left(\Delta W_{2}\right)^{2}\right\rangle \geq \frac{1}{64}\left|\left\langle\left[a_{1}, a_{1}^{+}\right]\right\rangle+\left\langle\left[a_{2}, a_{2}^{+}\right]\right\rangle\right|^{2}
$$

A state is said to be two-mode $q$-squeezed if

$$
\left\langle\left(\Delta W_{i}\right)^{2}\right\rangle<\frac{1}{8}\left|\left\langle\left[a_{1}, a_{1}^{+}\right]\right\rangle+\left\langle\left[a_{2}, a_{2}^{+}\right]\right\rangle\right| \quad(i=1 \text { or } 2) .
$$

For the even and odd $q$-deformed charge coherent states, the fluctuations are given by

$$
\begin{aligned}
e_{e(o)}\left\langle\left(\Delta W_{1}\right)^{2}\right\rangle_{e(o)}= & e_{(o)}\left\langle\left(\Delta W_{2}\right)^{2}\right\rangle_{e(o)}=\frac{1}{2}\left(_{e(o)}\left\langle\left(\Delta Y_{1}\right)^{2}\right\rangle_{e(o)}+e_{e(o)}\left\langle\left(\Delta Z_{1}\right)^{2}\right\rangle_{e(o)}\right) \\
= & \frac{1}{2}\left(_{e(o)}\left\langle\left(\Delta Y_{2}\right)^{2}\right\rangle_{e(o)}+e_{e(o)}\left\langle\left(\Delta Z_{2}\right)^{2}\right\rangle_{e(o)}\right) \\
= & \frac{1}{8}\left(_{e(o)}\left\langle\left[a_{1}, a_{1}^{+}\right]\right\rangle_{e(o)}+e_{e(o)}\left\langle\left[a_{2}, a_{2}^{+}\right]\right\rangle_{e(o)}+2_{e(o)}\left\langle a_{1}^{+} a_{1}\right\rangle_{e(o)}\right. \\
& \left.+2_{e(o)}\left\langle a_{2}^{+} a_{2}\right\rangle_{e(o)}\right) \\
> & \frac{1}{8}\left({ }_{e(o)}\left\langle\left[a_{1}, a_{1}^{+}\right]\right\rangle_{e(o)}+e_{e(o)}\left\langle\left[a_{2}, a_{2}^{+}\right]\right\rangle_{e(o)}\right)
\end{aligned}
$$


This shows there is no two-mode $q$-squeezing in both even and odd $q$-deformed charge coherent states. On the contrary, there is such $q$-squeezing in the $q$-deformed charge coherent states [61].

\subsection{Two-mode $q$-antibunching}

In analogy with the definition of two-mode antibunching [34], we introduce a two-mode $q$-correlation function as

$$
g \equiv \frac{\left\langle\left(a_{1}^{+} a_{2}^{+}\right)^{2}\left(a_{1} a_{2}\right)^{2}\right\rangle}{\left\langle a_{1}^{+} a_{2}^{+} a_{1} a_{2}\right\rangle^{2}}=\frac{\left\langle:\left(\left[N_{1}\right]\left[N_{2}\right]\right)^{2}:\right\rangle}{\left\langle\left[N_{1}\right]\left[N_{2}\right]\right\rangle^{2}},
$$

where $a_{i}$ and $a_{i}^{+}$represent the annihilation and creation operators of $q$-deformed photons of a deformed light field and : : denotes normal ordering. Physically, $g$ is a measure of $q$-deformed two-photon correlations in the $q$-deformed two-mode field and is related to the $q$-deformed two-photon number distributions. A state is said to be two-mode $q$-antibunched if

$$
g<1
$$

For the even and odd $q$-deformed charge coherent states, we have

$$
\begin{aligned}
& g_{e}=\overline{\operatorname{coth}}_{\underline{q}}{ }^{2}|\xi|^{2}, \\
& g_{o}=\overline{\tanh }_{\underline{q}}{ }^{2}|\xi|^{2} .
\end{aligned}
$$

From the above discussion about the function $\overline{\operatorname{coth}}_{\underline{q}}|\xi|^{2}\left(\overline{\tanh }_{\underline{q}}|\xi|^{2}\right)$, we see that $g_{e(o)}$ can be less than 1 over some particular range of $|\xi|$ values. This indicates that for both even and odd $q$-deformed charge coherent states, two-mode $q$-antibunching exists. On the contrary, for the $q$-deformed charge coherent states we have $g=1$ so that no two-mode $q$-antibunching exists. 
It can be shown that in the limit $q \rightarrow 1$, the nonclassical properties of the usual even and odd charge coherent states, studied in Ref. [34], are retrieved as expected.

\section{Summary}

Let us sum up the results obtained in the present Letter:

(1) The even and odd $q$-deformed charge coherent states, defined as the two orthonormalized eigenstates of both the square of the pair $q$-boson annihilation operator and the charge operator, have been constructed and their (over)completeness proved. Such $q$-deformed states become the usual even and odd charge coherent states in the limit $q \rightarrow 1$.

(2) The even (odd) q-deformed charge coherent states have been shown to be generated by the symmetric (antisymmetric) combination of $q$-deformed charge coherent states in the case of conserved charge, or by a suitable average over the $U(1)$-group (caused by the charge operator) action on the product of $q$-deformed coherent states and even (odd) q-deformed coherent states.

(3) The $D$-algebra of the $\mathrm{SU}_{q}(1,1)$ generators corresponding to the even and odd $q$-deformed charge coherent states has been realized in a $q$-differential operator matrix form.

(4) Both even and odd $q$-deformed charge coherent states have been shown to exhibit $\mathrm{SU}_{q}(1,1)$ squeezing and two-mode $q$-antibunching, but neither single-mode nor two-mode $q$-squeezing. 


\section{Acknowledgments}

X.-M.L. is grateful to Professor C. Quesne for warm hospitality at the Université Libre de Bruxelles. He also acknowledges financial support by the National Fund for Scientific Research (FNRS), Belgium, as well as support by the National Natural Science Foundation of China under Grants Nos. 10174007, 10074008 and 60278021. C.Q. is a Research Director of the National Fund for Scientific Research (FNRS), Belgium. 


\section{References}

[1] E. Schrödinger, Naturwissenschaften 14 (1926) 664.

[2] R.J. Glauber, Phys. Rev. A 131 (1963) 2766.

[3] J.R. Klauder and E.C.G. Sudarshan, Fundamentals of Quantum Optics, Benjamin, New York, 1968.

[4] J.R. Klauder and B.S. Skagerstam, Coherent States - Applications in Physics and Mathematical Physics, World Scientific, Singapore, 1985.

[5] A. Perelomov, Generalized Coherent States and Their Applications, Springer, Berlin, 1986.

[6] W.M. Zhang, D.H. Feng and R. Gilmore, Rev. Mod. Phys. 62 (1990) 867.

[7] S.T. Ali, J.-P. Antoine and J.-P. Gazeau, Coherent States, Wavelets and Their Generalizations, Springer, New York, 2000.

[8] D. Bhaumik, K. Bhaumik and B. Dutta-Roy, J. Phys. A 9 (1976) 1507.

[9] K.E. Eriksson and B.S. Skagerstam, J. Phys. A 12 (1979) 2175; J. Phys. A 14 (1981) $545(\mathrm{E})$.

[10] B.S. Skagerstam, Phys. Lett. A 69 (1978) 76.

[11] H.Y. Fan and T.N. Ruan, Commun. in Theor. Phys. (Beijing, China) 2 (1983) 1405 .

[12] B.S. Skagerstam, J. Phys. A 18 (1985) 1.

[13] D. Horn and R. Silver, Ann. Phys. (N.Y.) 66 (1971) 509.

[14] K.E. Eriksson, N. Mukunda and B.S. Skagerstam, Phys. Rev. D 24 (1981) 2615.

[15] J.C. Botke, D.J. Scalapino and R.L. Sugar, Phys. Rev. D 9 (1974) 813.

[16] M. Martinis and V. Mikuta, Phys. Rev. D 12 (1975) 909.

[17] M. Bosterli, Phys. Rev. D 16 (1977) 1749.

[18] B.S. Skagerstam, Phys. Rev. D 19 (1979) 2471; Phys. Rev. D 22 (1980) 534(E).

[19] G.J. Ni and Y.P. Wang, Phys. Rev. D 27 (1983) 969.

[20] P. Chattopadhyay and J. Da Providencia, Nucl. Phys. A 370 (1981) 445.

[21] B.S. Skagerstam, Z. Phys. C 24 (1984) 97.

[22] B.S. Skagerstam, Phys. Lett. B 133 (1983) 419.

[23] A.T.M. Hertz, T.H. Hansson and B.S. Skagerstam, Phys. Lett. B 145 (1984) 123.

[24] M.M. Nieto, Phys. Rev. D 30 (1984) 770. 
[25] G.S. Agarwal, Phys. Rev. Lett. 57 (1986) 827.

[26] G.S. Agarwal, J. Opt. Soc. Am. B 5 (1988) 1940.

[27] C.C. Gerry, J. Mod. Opt. 42 (1995) 585.

[28] S.C. Gou, J. Steinbach and P.L. Knight, Phys. Rev. A 54 (1996) R1014.

[29] S.B. Zheng and G.C. Guo, Quantum Semiclass. Opt. 10 (1998) 441.

[30] V.V. Dodonov, I.A. Malkin and V.I. Man'ko, Physica 72 (1974) 597.

[31] M. Hillery, Phys. Rev. A 36 (1987) 3796.

[32] Y.J. Xia and G.C. Guo, Phys. Lett. A 136 (1989) 281.

[33] V. Bužek, A. Vidiella-Barranco and P.L. Knight, Phys. Rev. A 45 (1992) 6750.

[34] Xiao-Ming Liu, Phys. Lett. A 279 (2001) 123.

[35] V.G. Drinfeld, Quantum Groups, in: A.M. Gleason (Ed.), Proc. Int. Congr. of Math., Berkeley, vol. I, (American Mathematicial Society, Providence, RI, 1986) p. 798 .

[36] M. Jimbo, Lett. Math. Phys. 10 (1985) 63.

[37] L.C. Biedenharn, J. Phys. A 22 (1989) L873.

[38] M. Chaichian, D. Ellinas and P. Kulish, Phys. Rev. Lett. 65 (1990) 980.

[39] P.P. Kulish and E.V. Damaskinsky, J. Phys. A 23 (1990) L415.

[40] C. Quesne, Phys. Lett. A 153 (1991) 303.

[41] B. Jurčo, Lett. Math. Phys. 21 (1991) 51.

[42] A.J. Macfarlane, J. Phys. A 22 (1989) 4581.

[43] C.P. Sun and H.C. Fu, J. Phys. A 22 (1989) L983.

[44] R.W. Gray and C.A. Nelson, J. Phys. A 23 (1990) L945.

[45] A.J. Bracken, D.S. McAnally, R.B. Zhang and M.D. Gould, J. Phys. A 24 (1991) 1379 .

[46] A.I. Solomon and J. Katriel, J. Phys. A 23 (1990) L1209.

[47] E. Celeghini, M. Rasetti and G. Vitiello, Phys. Rev. Lett. 66 (1991) 2056.

[48] V. Bužek, J. Mod. Opt. 38 (1991) 801.

[49] E.G. Floratos, J. Phys. A 24 (1991) 4739.

[50] S.H. Chiu, R.W. Gray and C.A. Nelson, Phys. Lett. A 164 (1992) 237.

[51] H.Y. Fan and C.P. Sun, Commun. Theor. Phys. 17 (1992) 243. 
[52] S. Chaturvedi and V. Srinivasan, Phys. Rev. A 44 (1991) 8020.

[53] F.B. Wang and L.M. Kuang, Phys. Lett. A 169 (1992) 225.

[54] X.C. Song, J. Phys. A 23 (1990) L821.

[55] H. Exton, $q$-Hypergeometric Functions and Applications, Ellis Horwood, Chichester, 1983, p. 172.

[56] K.H. Cho, Chaiho Rim, D.S. Soh and S.U. Park, J. Phys. A 27 (1994) 2811.

[57] G.E. Andrews, $q$-Series: Their Development and Application in Analysis, Number Theory, Combinatorics, Physics, and Computer Algebra, Conference Board of the Mathematical Sciences 66, American Mathematical Society, Providence, RI, 1986.

[58] D. Bernard and A. Leclair, Phys. Lett. B 227 (1989) 417.

[59] R. Gilmore, C.M. Bowden and L.M. Narducci, Phys. Rev. A 12 (1975) 1019.

[60] M. Haken, Handbuch der Physik, vol. XXV/2, Springer, Berlin, 1970.

[61] K. Wódkiewicz and J.H. Eberly, J. Opt. Soc. Am. B 2 (1985) 458.

[62] X.-M. Liu, unpublished.

[63] R. Loudon and P.L. Knight, J. Mod. Opt. 34 (1987) 709. 